\title{
Programação de Computadores e Robótica Educativa na Escola: tendências evidenciadas nas produções do Workshop de Informática na Escola
}

\author{
Valéria Espíndola Lessa ${ }^{1}$, Franciele Meinerz Forigo², Adriano Canabarro \\ Teixeira $^{3}$, Gabriel Paludo Licks ${ }^{4}$ \\ ${ }^{1}$ Instituto Federal do Rio Grande do sul - Erechim - RS - Brazil \\ ${ }^{2}$ Instituto Federal Farroupilha - Santa Rosa - RS - Brazil \\ ${ }^{3,4}$ Universidade de Passo Fundo (UPF) - Passo Fundo - RS - Brazil \\ \{lessavaleria@gmail.com, francieleforigo@gmail.com, teixeira@upf.br, \\ gabrielplicks@gmail.com\}
}

\begin{abstract}
This paper presents an analysis of the main trends in computer programming and educational robotics in school, shown in the scientific production from the Workshop de Informática na Escola, in ten editions of the event, held during the years of 2005 to 2014. The work takes a quantitative and qualitative approach, from which we identified the characteristics of the productions published in the annals of that event, as well as the software and hardware used and the pedagogical purposes, which puts in evidence different goals in the use of technology. The study shows that, in face of development of technologies, some initial advances have been made.
\end{abstract}

Resumo. Este texto apresenta uma análise sobre as principais tendências em programação de computadores e em robótica educativa na escola, evidenciadas nas produções cientificas do Workshop de Informática na Escola, nas dez edições do evento, realizadas de 2005 a 2014. O trabalho adota uma abordagem quanti-qualitativa, a partir da qual identificamos as características das produções publicadas nos anais do referido evento, assim como os softwares e hardwares utilizados, as intenções pedagógicas, as quais evidenciam distintos objetivos de uso das tecnologias. O estudo mostra que em face do desenvolvimento das tecnologias alguns avanços têm sido alcançados de forma incipiente.

\section{Introdução}

Os desenvolvimentos das tecnologias digitais têm influenciado mudanças nos modos de comunicação, de acesso às informações e, consequentemente, em relação ao saber da sociedade contemporânea (LEVY, 2010). Apresenta-se como um potencial de modificação do processo educativo ao proporcionar o desenvolvimento de habilidades e atitudes para se viver em um mundo cada vez mais globalizado. Para Lucena (1997), a tecnologia fundamenta um estilo educacional em busca de um novo paradigma, pelo qual o aluno tem possibilidades de desenvolver estruturas lógicas, raciocínio crítico e capacidade de decisão, preparando-se para uma nova sociedade. 
As tecnologias trazem potencialidades que precisariam ser direcionadas no sentido da emancipação (FREIRE, 2005) dos seus usuários, indo de encontro ao consumo passivo de softwares mais complexos a cada dia, na qual temos pouco entendimento e pouco controle, conduzindo-nos a cada vez menos poder (RUSHKOFF, 2010). Segundo o autor, "programação é o ponto de impacto, o ponto de apoio a alavancagem significativa em uma sociedade digital. Se não aprendermos a programar, arriscamos a ser programados.” (p.142).

Seymour Papert $(1985,2008)$ desenvolveu pesquisas no Instituto de Tecnologia de Massachusetts (MIT), na qual a programação de computadores é apresentada como uma possibilidade de permitir às crianças a criação de um mundo digital criativo, cheio de conceitos de matemática, de física e de outras áreas do conhecimento, onde ela se torna um agente ativo de sua aprendizagem. Para ele, a aprendizagem está relacionada à ação de inventar e, no processo de criar a programação, a criança concretiza na linguagem do software conhecimentos que muitas vezes são abstratos e de difícil compreensão para ela.

A robótica, sendo um aparato tecnológico que demanda programação, agrega, às características acima apresentadas, a possibilidade de materialização daquilo que, até então, era visível somente na tela do computador. Outra característica da robótica educacional é o fato de suas atividades serem mais produtivas quando realizadas por um grupo de pessoas que trabalham em conjunto e não por um único indivíduo. Ao congregar a teoria à prática, ela é capaz de desenvolver nos alunos algumas habilidades que a dinâmica das demais disciplinas quase não aborda como: trabalho em equipe, colaboração, autodesenvolvimento, capacidade de solucionar problemas, senso crítico, integração de disciplinas, exposição de pensamentos, argumentação, criatividade, autonomia e responsabilidade, postura empreendedora, entre outras. Por se tratar de uma área multidisciplinar, a robótica estimula os alunos a buscarem soluções que integram conceitos e aplicações de outras disciplinas envolvidas, como matemática, física, mecânica, eletrônica, design e informática.

Neste sentido, acreditamos que não basta apenas ter computadores nas escolas e dizer que o processo educativo concretizou-se com apoio da tecnologia. Da mesma forma que não basta utilizar as tecnologias na sala de aula de forma mecânica que privilegia a continuidade de uma metodologia "bancária" de ensino (FREIRE, 2005). Surge, então, um ponto crucial nesse contexto: que ações envolvendo a programação de computadores e a robótica educativa são realizadas nas escolas de forma a qualificar o processo de aprendizagem?

$\mathrm{Na}$ esteira deste movimento são promovidos diversos eventos que se ocupam da temática, dentre eles o Workshop de Informática na Escola (WIE), promovido pela Comissão Especial de Informática na Educação (CEIE) da Sociedade Brasileira de Informática (SBC), que ocorre anualmente desde 1995. Os trabalhos submetidos ao WIE possuem caráter aplicado, como relato de experiências ou pesquisas desenvolvidas nos espaços escolares com o uso de tecnologias ${ }^{1}$.

\footnotetext{
${ }^{1}$ http://ic.ufal.br/evento/cbie_laclo2015/eventos\#WIE
} 
Neste sentido, diferentes ações são desenvolvidas e divulgadas sobre a utilização de tecnologias digitais na escola, tais como a utilização de programação de computadores e robótica educativa com objetivos, enfoques, softwares e hardwares variados. Isto posto e considerando a relevância do WIE para o desenvolvimento e concretização da informática na escola, realizamos um estudo, de natureza quantiqualitativa, que busca evidenciar as principais dimensões sobre a programação de computadores e robótica na escola contemplados nos trabalhos apresentados em dez edições do evento, de 2005 a 2014, a partir da publicação on-line ${ }^{2}$ dos anais.

Este estudo é realizado por pesquisadores do Grupo de Inclusão Digital (GEPID) da Universidade de Passo Fundo, cuja temática de programação de computadores e de robótica estão presentes nas discussões e pesquisas de iniciação científica, mestrado e doutorado do grupo. Organizado em trono de duas linhas de pesquisa, Estudos da Cibercultura e Tecnologias e Metodologias de Inclusão Digital, o GEPID busca estudar e pesquisar tecnologias, metodologias e ações de inclusão digital que busquem qualificar a informática educativa no contexto educacional brasileiro através de atividades científicas e de extensão que envolvam, dentre outras áreas, a Robótica Educacional Livre e a Programação para Crianças.

A metodologia de trabalho procedeu à análise qualitativa, segundo critérios definidos como de maior relevância ao estudo, seguido de levantamento quantitativo destes critérios e na representação gráfica no âmbito do WIE. Os critérios utilizados foram: número total de trabalhos publicados em 10 anos de evento; número de trabalhos envolvendo a temática deste estudo (programação ou robótica) por ano, por região do país, por nível de ensino, por instrumento tecnológico utilizado (softwares e hardwares) e por intenções pedagógicas percebidas ${ }^{3}$. As etapas do estudo compreenderam a leitura de cada trabalho relacionado ás temáticas alvo nos 10 anos de evento, seguido de uma classificação quanto aos critérios definidos, sendo o último realizado após uma leitura mais cuidadosa de cada uma das propostas sobre programação de computadores e robótica educativa.

\section{Workshop de Informática na Escola}

O WIE teve sua primeira edição no ano de 1995 e até 2010 era um evento científíco realizado junto com o Congresso da Sociedade Brasileira de Computação (CSBC), a cada ano numa sede diferente, chamado também de evento satélite. A partir de 2011, o WIE passou a integrar o Congresso Brasileiro de Informática na Educação (CBIE), no qual os objetivos continuam sendo os de divulgar ações envolvendo tecnologias aplicadas ao processo de ensino e aprendizagem na escola e de proporcionar um espaço de discussão e debate de ideias em torno do tema.

Os trabalhos são submetidos em três grupos diferentes de discussão, chamados trilhas, na qual se constituem, no ano de 2015, em: Trilha 1: Relatos de Experiência de uso de Tecnologias Digitais de Informação e Comunicação (TDIC) na Educação; Trilha 2: Formação de Recursos Humanos para o Uso das Tecnologias Digitais de

\footnotetext{
2 http://www.br-ie.org/pub/index.php/wie/index

${ }^{3}$ A planilha com os dados pode ser visualizada em https://goo.gl/WCxghO
} 
Informação e Comunicação (TDIC) na Educação; Trilha 3: Impactos das Tecnologias Digitais de Informação e Comunicação (TDIC) na Sociedade.

A partir de um levantamento de dados sobre os trabalhos que compõe os anais de dez anos do WIE, apuramos que foram publicados artigos completos, com até 10 páginas e artigos resumidos, com até 5 páginas, todos inseridos nas temáticas das trilhas. Neste conjunto de trabalhos, importantes dimensões da programação de computadores e da robótica foram contempladas, conforme será discutido no quarto tópico. A fim de analisar de uma forma global a produção científica submetida ao WIE, tabulamos o número total destes trabalhos submetidos nos anos de 2005 a 2014, para posteriormente localizar os que tratam das temáticas de interesse deste artigo.

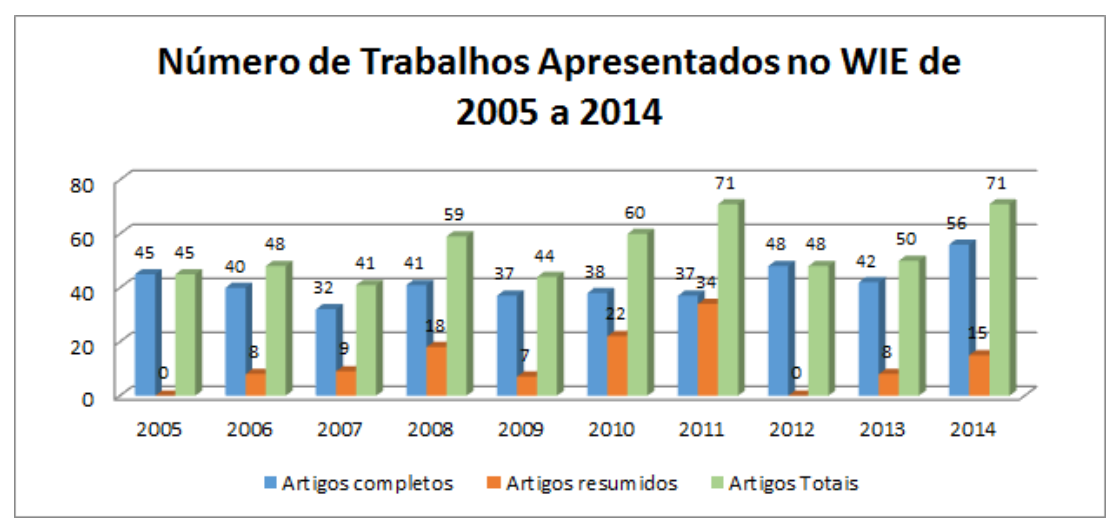

Figura 1. Evolução do número de trabalhos publicados nos anais de 10 anos do WIE.

Conforme o gráfico representado na Figura 1, vemos um crescimento no número total de trabalhos de 2005 para 2006, seguido de uma diminuição em 2007 voltando a crescer em 2008. Em 2009, 2010 e 2011 temos novamente uma diminuição seguida de um aumento. E o mesmo ocorre em 2012, 2013 e 2014. Os anos de 2011 e 2014 receberam o maior número de trabalhos, totalizando $71 \mathrm{em}$ cada e é possível observar que há uma média aproximada de 54 trabalhos por evento, nestes dez anos. Também observa-se um maior número de trabalhos completos do que resumidos.

De qualquer forma, ao se analisar especificamente os trabalhos completos, que geralmente se referem a pesquisas finalizadas, é possível verificar que tem uma variação de 32 a 42 artigos em 8 das edições do evento, sendo que em somente duas, a de 2012 e 2014, existe um aumento sensível no número de trabalhos completos. A partir destes dados, vemos que existe um número similar e constante de trabalhos apresentados no evento ao longo dos dez anos, porém acreditamos não ser possível inferir sobre o crescimento do número de pesquisas e experiências no país. Para isso, seria necessário um estudo mais aprofundado a respeito dos trabalhos submetidos à avaliação no evento e não apenas dos selecionados, o que foge da proposta deste artigo.

\section{Discussões sobre Programação de Computadores e Robótica}

Enquanto alternativa para qualificação da informática educativa, a robótica e a programação não são novidade. Tal constatação passa pelo reconhecimento das 
discussões realizadas por Papert (1985) já na década de 60 e é corroborada nas produções realizadas no decorrer das diferentes edições do WIE, foco da análise deste artigo. Entretanto, é preciso reconhecer que dado o potencial destas possibilidades didáticas baseadas em tecnologia, com a criação de ambientes propícios ao desenvolvimento de habilidades cognitivas que podem ter profundos desdobramentos em outras áreas do conhecimento, a temática ainda é pouco explorada.

A programação e a robótica proporcionam ao aprendiz um ambiente desafiador que o estimula a pensar. Isso pressupõe um processo de aprendizagem ativa, onde a construção do conhecimento se dá a partir das ações físicas ou mentais do aluno. Programar envolve uma série de capacidades, das quais destacamos: criatividade, capacidade de resolução de problemas, trabalho em grupo e o raciocínio lógico. A robótica por sua vez, proporciona a materialização dos resultados da programação, agregando outras áreas do conhecimento para sua concretização, assumindo "o papel de uma ponte de ligação interdisciplinar visando a construção do conhecimento coletivo através da aplicação com a realidade. " (Trentin; Pérez; Teixeira. 2013).

\section{Análise do levantamento}

Para nossas análises tomamos como base os artigos completos e resumidos publicados nas dez edições do WIE, realizados de 2005 a 2014, num total de 537 artigos. Ao buscar os trabalhos que contemplassem os temas de nosso estudo, encontramos $19(3,5 \%)$ sobre a programação de computadores e $15(2,8 \%)$ sobre robótica, evidenciando um baixo percentual de trabalhos dos referidos temas. Além disso, observamos a evolução do aparecimento dos temas ao longo dos dez anos, conforme o gráfico representado da Figura 2, no qual é possível perceber um aumento de sua incidência nos últimos 5 anos. Acreditamos que, diferentemente da análise feita sobre o WIE como um todo, a ampliação do número de artigos que tratam das temáticas alvo deste artigo, deve-se à popularização dos softwares e hardwares utilizados, bem como, à crescente facilidade de uso destes recursos.

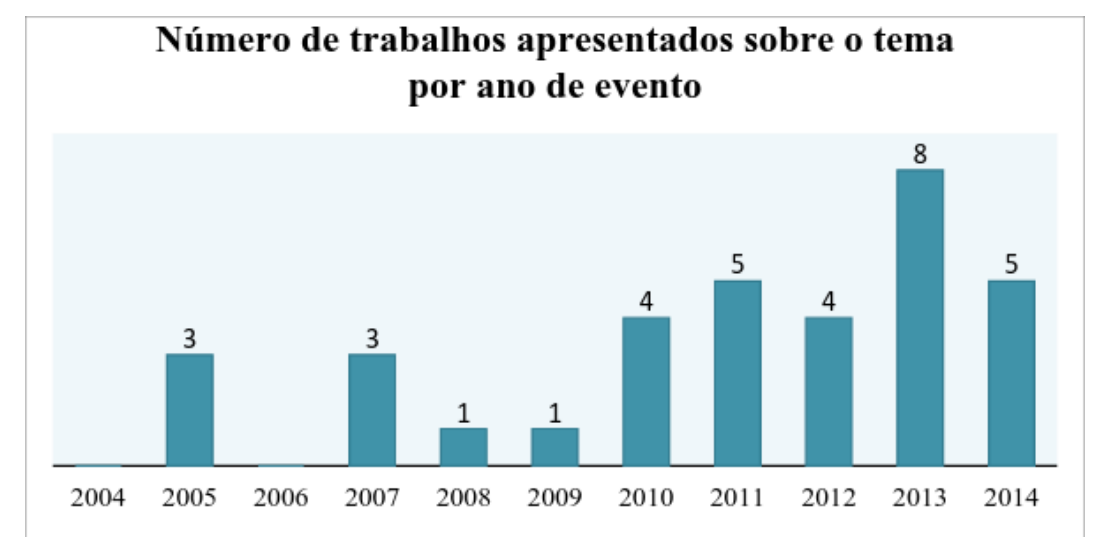

Figura 2. Levantamento contendo o número de trabalhos apresentados no WIE sobre programação e robótica por ano de evento, de 2005 a $2014(n=34)$.

Sob outra ótica, também levantamos o número de publicações de artigos sobre a temática por região do país, conforme o mapa A, da Figura 3. Os mapas B e C trazem de forma separada a quantidade de artigos, também por região, sobre programação de 
computadores e robótica, respectivamente. Assim vemos maior incidência de trabalhos voltados à programação de computadores na região nordeste, e de robótica na região sudeste. Juntos, os trabalhos se concentram mais na região nordeste.

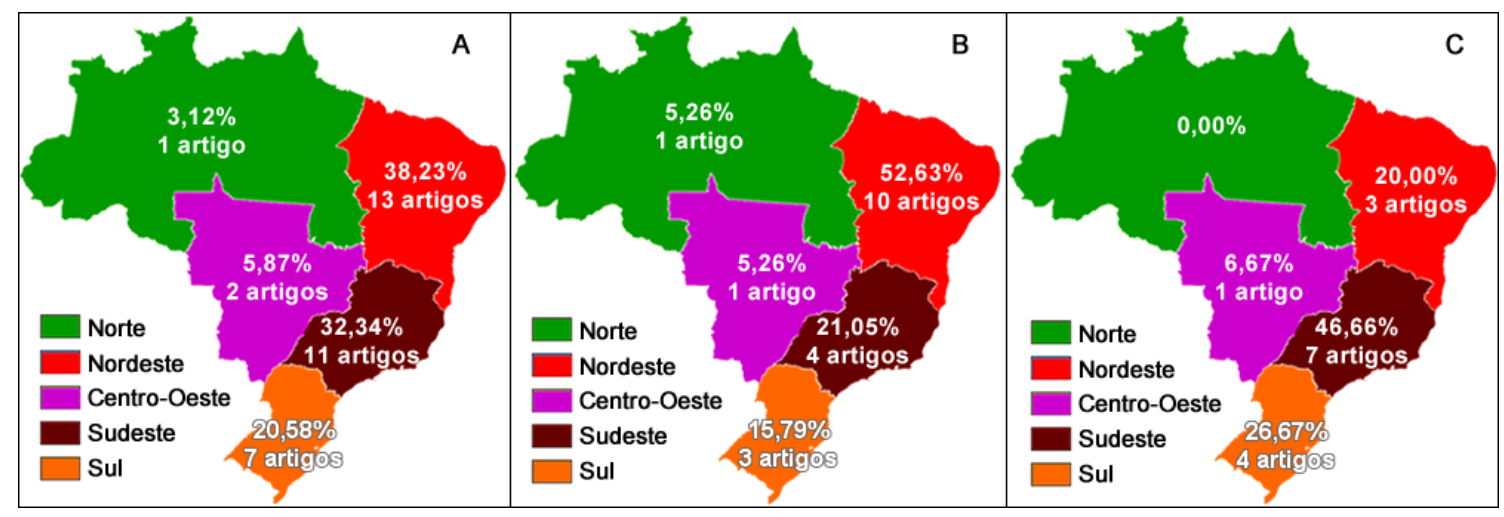

Figura 3. Levantamento dos trabalhos apresentados no WIE sobre programação e robótica por região do país, de 2005 a $2014(n=34)$.

Realizando uma análise mais detalhada das regiões que apresentaram maior número de artigos publicados dentro da temática da Programação de Computadores e da Robótica Educativa, regiões Nordeste e Sudeste respectivamente, identificamos que são provenientes eminentemente de Instituições de Ensino Superior públicas, com destaque para a Universidade Federal da Paraíba, com 4 artigos na área de Programação e a Universidade Federal do Rio de Janeiro, com 2 artigos na área de robótica, diferença não muito significativa na temática. Outro aspecto a ser destacado, desta vez considerando toda a produção nestas duas áreas, é o fato de que existe somente um artigo publicado por uma universidade que não é pública, a Universidade de Passo Fundo no Rio Grande do Sul que se enquadra na categoria de Universidade Comunitária.

Dando continuidade às análises dos conteúdos destes 34 artigos, optamos por focar em três aspectos que consideramos relevantes para nossas pesquisas no GEPID: (i) nível de ensino de realização da ação; (ii) hardwares e softwares utilizados; (iii) intenções pedagógicas evidenciadas nas propostas. Para tanto, realizamos leituras de todos os artigos e os organizamos a partir destas categorias de análise.

Quanto ao nível de ensino, percebe-se, como era de se esperar em trabalhos submetidos ao WIE, a incidência de trabalhos realizados junto a escolas da educação básica, com 11 ações para o ensino fundamental e 12 para o ensino médio. Ao se analisar como se dá a distribuição das temáticas investigadas neste estudo por nível de ensino, é possível verificar uma equivalência entre robótica e programação no ensino fundamental e médio. Entretanto, um dado interessante se revela ao se analisar de forma longitudinal esta produção. De 2005 a 2010, os artigos eram predominantemente de aplicações de robótica, totalizando 9 em relação aos 3 envolvendo programação de computadores. A partir de 2011, no entanto, esta relação se inverte apresentando 15 artigos sobre programação e somente 6 de robótica.

Isto evidencia que está havendo um movimento de preocupação com a educação da escola básica e que as iniciativas inovadoras, como o caso da programação e da 
robótica, estão acontecendo, mesmo que de forma inicial, conforme pode ser observado na figura seguinte.

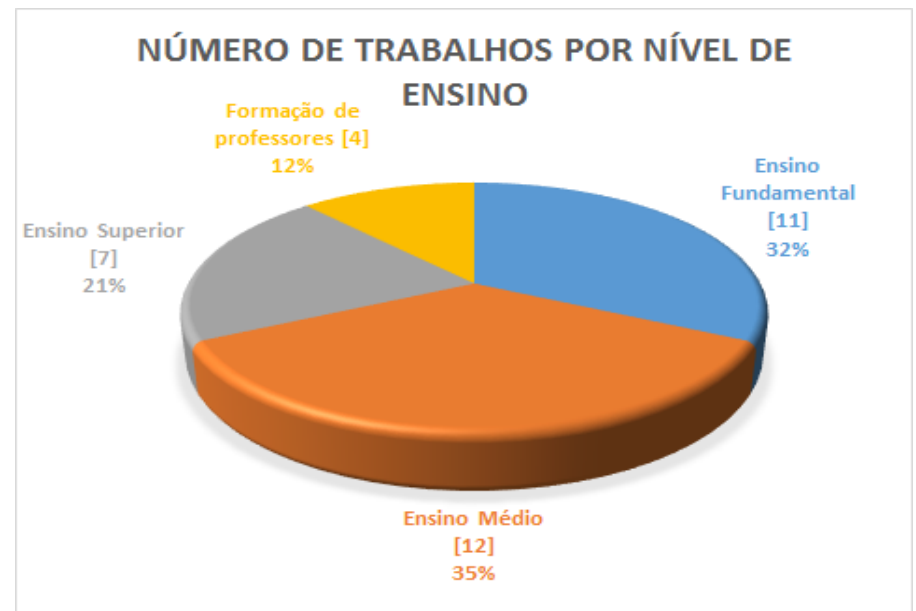

Figura 4. Número de trabalhos do WIE envolvendo programação de computadores e robótica por nível de ensino $(n=34)$.

O gráfico da Figura 4 aponta para outra situação importante quando se pensa em consolidação de uma área de pesquisa enquanto prática educativa que acontece na escola sem a intervenção de Instituição de Ensino Superior: a formação docente, categoria que apresenta somente $12 \%$ dos trabalhos realizados. É preciso que as pesquisas científicas ampliem sua interação com os professores a fim de que os conhecimentos gerados na academia possam ganhar força e escala dentro da educação formal.

Em relação das ferramentas utilizadas nos trabalhos, percebemos softwares e hardwares variados. Observamos aproximadamente 21 softwares diferentes, sendo que o Scratch $^{4}$ foi o mais frequente em relação aos demais, utilizado em 6 (18\%) dos 34 trabalhos analisados. Sobre os hardwares utilizados, dos 15 trabalhos de robótica, percebemos que 8 utilizaram a placa Arduino associada a componentes eletrônicos e 7 utilizaram kits robóticos ${ }^{5}$ como LEGO Mindstorms, GoGo Board e Hajime.

Embora o percentual de $18 \%$ de pesquisas utilizando o Scratch não seja significativo isoladamente, comparando-se com o número total de softwares, a solução criada pelo Instituto de Tecnologia de Massachussets (MIT) destaca-se como alternativa poderosa para a educação. Entretanto, ao se analisar o percentual dos artigos sobre Robótica que relatam a utilização do Arduino, identificamos que a metade optou por esta tecnologia. Vale ressaltar que tanto o Scratch quanto o Arduino são tecnologias livres que dão conta da produção do conhecimento, com desempenho igual ou superior às tecnologias proprietárias, geralmente pagas.

\footnotetext{
${ }^{4}$ O software de programação Scratch, foi elaborado no Massachusetts Institute of Tecnology (MIT http:// http://web.mit.edu/) por uma equipe de pesquisadores liderados por Mitchel Resnick.

${ }^{5}$ Os kits de robótica são compostos por peças destinadas para montagem de objetos robóticos e por um software que possibilita a programação. Os kits podem ser comerciais ou de robótica livre.
} 
Quanto às intenções pedagógicas, procuramos evidenciar o foco principal da abordagem da temática e foi possível verificar quatro categorias: (i) ensino de programação/robótica em si; (ii) ensino de conteúdos escolares específicos através da programação/robótica; (iii) formação de professores; e (iv) projetos visando a acessibilidade econômica para o uso de robótica. Os resultados podem ser visualizados na Figura 5.

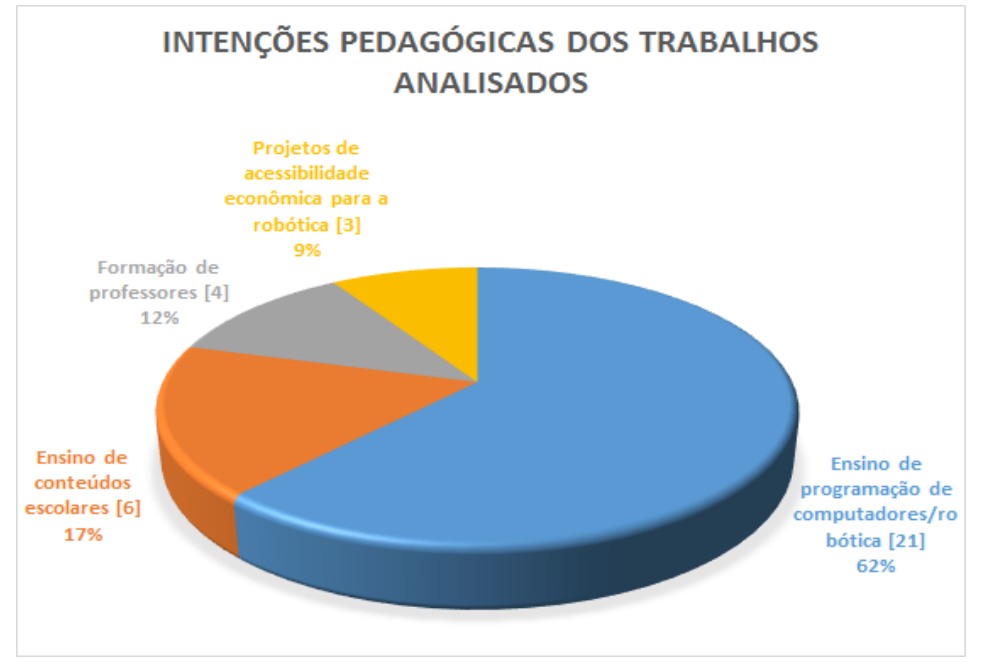

Figura 5. Levantamento sobre as intenções pedagógicas dos 34 trabalhos analisados.

Além da percepção que a maioria dos artigos (62\%), visam o ensino da programação e da robótica em si, sem o foco de ensinar conteúdos específicos do currículo escolar, no cruzamento destes dados com o nível de ensino, encontramos que 15 destes 21 trabalhos estão voltados ao ensino médio e fundamental, evidenciando a inclusão de conteúdos de informática, a priori de nível superior, na escola básica. Assim, os referidos artigos divulgaram ações que objetivavam proporcionar $\mathrm{o}$ desenvolvimento de habilidades e capacidades importantes para os estudantes como criatividade e autonomia. Tal constatação vai à contramão da concepção de tecnologia como meio para aprender conteúdos curriculares o que, de certa forma, parece uma tendência macro da área e o que aponta para a demanda de acompanhamento dos desdobramentos de ações deste tipo sobre a vida acadêmica dos alunos e em outras dimensões de sua escolarização.

Os seis trabalhos que focaram no ensino de conteúdos específicos por meio da programação/robótica (17\%), envolveram conteúdos de matemática, física, química, geografia, português e educação ambiental, e foram experiências realizadas na educação básica. É importante destacar este ponto de nossa análise pois Papert (1985) já argumentava em seu livro Logo, que a programação de computadores até pode colaborar no ensino de certos conteúdos escolares, mas ele a enxerga como forma de aprendizagem dissociada de currículo, na qual a criança aprenderá aquilo que ela necessitar ao criar o que lhe dá entusiasmo. Portanto, ao delimitar conteúdos específicos do currículo escolar no trabalho com a programação e robótica, talvez estejamos limitando as possibilidades de criação dos estudantes. Ainda, é possível apontar que a distribuição dos trabalhos entre aplicações em conteúdos escolares (17\%) e 
aprendizagem da tecnologia em si (62\%), atende à premissa apresentada por Papert a 30 anos atrás.

Existem três trabalhos sobre acessibilidade econômica para robótica que não chegaram a ser aplicados, o que nos leva a caracterizá-los como projetos. No entanto, são trabalhos importantes, tendo em vista o alto custo financeiro para escolas públicas adquirirem os kits robóticos necessários para as ações deste tipo. Dessa forma, estes trabalhos mostram possibilidades da robótica como ferramenta educacional de custo reduzido a partir do uso de materiais reciclados e de sucata.

Sobre a formação de professores, encontramos apenas três trabalhos, dois com professores da educação básica e apenas um com estudantes de licenciatura. O WIE possui grande interesse neste critério, pois, conforme já foi apresentado, a trilha 2 tem foco na formação de recursos humanos para o uso de tecnologias na educação. No entanto, não chegamos a analisar os trabalhos a partir das trilhas temáticas do evento e, assim, não temos a dimensão das discussões que estão sendo feitas neste sentido. De todo modo, os referidos artigos trazem preocupações sobre a realidade da formação de professores em tecnologias e apresentam experiências na qual a programação e a robótica são vistas como ferramentas auxiliares a fim de qualificar a prática docente. Embora represente um avanço, mostra uma carência importante para a informática educativa na efetivação dos conhecimentos gerados no interior dos grupos de pesquisa nas escolas.

\section{Considerações Finais}

A programação de computadores e a robótica educativa não são temas novos no campo educacional, porém carecem de mais estudos e ações voltadas às escolas. A partir de nossas análises sobre os artigos completos e resumidos dos 10 últimos anos do WIE (2005 a 2014) foi possível perceber como ainda é incipiente a divulgação de ações pedagógicas envolvendo a temática estudada. No entanto, foi possível apresentar um panorama dos trabalhos do evento a partir de nossos critérios e acreditamos que este estudo fornece subsídios para futuras pesquisas na área.

Levando em consideração o desenvolvimento acelerado dos aparatos tecnológicos no mundo contemporâneo e o fato de os temas pesquisados não serem inéditos, tendo em vista as pesquisas de Papert realizadas há mais de 50 anos, as ações inovadoras das escolas são pontuais e geralmente estão relacionadas com pesquisas realizadas em universidades. Dessa forma, é possível propor uma questão norteadora de pesquisa futura: qual é a situação da formação básica e continuada de professores na área de programação de computadores e robótica educativa no Brasil?

Nesse sentido, compreendemos que a concretização de ações envolvendo a programação de computadores e de robótica educativa na escola básica pressupõe, a priori, pesquisas científicas aplicadas, processos de formação continuada de professores e a divulgação e discussão do que é realizado por meio de eventos científicos como o WIE. Ou seja, é preciso que as novas tecnologias sejam pensadas de forma a qualificar o fazer pedagógico e que estes estudos cheguem à escola.

\section{Referências}

LEVY, Pierre. Cibercultura. 3.ed. São Paulo: Ed.34, 2010. 
LUCENA, Marisa. Um modelo de escola aberta na internet kidlink no Brasil. Rio de Janeiro: Brasport, 1997.

PAPERT, Seymour. A máquina das crianças: repensando a escola na era da informática. Ed.rev. Porto Alegre: Artmed, 2008.

PAPERT, Seymour. Logo: computadores e educação. Tradução de José A. Valente, Beatriz Bitelman e Afira V. Ripper. São Paulo: Editora Brasiliense, 1985.

RUSHKOFF, Douglas. As 10 questões essenciais da era digital: programa seu futuro para não ser programado por ele. São Paulo: Saraiva, 2012.

FREIRE, Paulo. Pedagogia do Oprimido. Rio de Janeiro: Paz e Terra, 2005.

TRENTIN, M. A., PÉREZ, C. A. S., TEIXEIRA, A. C. A robótica livre no auxílio da aprendizagem do movimento retilíneo. In: XIX Workshop de Informática na Escola. Campinas, SP. Unicamp, 2013. p. 51 a 59. 